\title{
Tubulin and actin interplay at the T cell and antigen-presenting cell interface
}

\author{
Noa Beatriz Martín-Cófreces ${ }^{1,2}$, Balbino Alarcón ${ }^{3}$ and Francisco Sánchez-Madrid ${ }^{1,2}$ * \\ ${ }^{1}$ Departamento de Biología Vascular e Inflamación, Centro Nacional de Investigaciones Cardiovasculares, Madrid, Spain \\ ${ }^{2}$ Servicio de Inmunología, Hospital Universitario de la Princesa, Universidad Autónoma de Madrid, Instituto Investigación Sanitaria Princesa, Madrid, Spain \\ ${ }^{3}$ Centro de Biología Molecular Severo Ochoa, Madrid, Spain
}

\section{Edited by:}

Takashi Saito, Research Center for

Allergy and Immunology, Japan

\section{Reviewed by:}

Xose R. Bustelo, Consejo Superior de Investigaciones Científicas, Spain

Ruggero Pardi, Vita-Salute San

Raffaele University, Italy

*Correspondence:

Francisco Sánchez-Madrid, Laboratorio de Comunicación Intercelular, Servicio de Inmunología, Hospital de La Princesa, Instituto de Investigación Sanitaria Princesa, Planta 1, Diego de León 62, 28006

Madrid, Spain.

e-mail: fsanchez.hlpr@salud.

madrid.org
T cells reorganize their actin and tubulin-based cytoskeletons to provide a physical basis to the immune synapse. However, growing evidence shows that their roles on $T$ cell activation are more dynamic than merely serving as tracks or scaffold for different molecules. The crosstalk between both skeletons may be important for the formation and movement of the lamella at the immunological synapse by increasing the adhesion of the $T$ cell to the antigen-presenting cells (APC), thus favoring the transport of components toward the plasma membrane and in turn regulating the T-APC intercellular communication. Microtubules and F-actin appear to be essential for the transport of the different signaling microclusters along the membrane, therefore facilitating the propagation of the signal. Finally, they can also be important for regulating the endocytosis, recycling, and degradation of the $T$ cell receptor signaling machinery, thus helping both to sustain the activated state and to switch it off.

Keywords: T cell, immune synapse, microtubule-organizing centre, actin

\section{INTRODUCTION}

The process of $\mathrm{T}$ cell activation by antigen-presenting cells (APC) that display a peptide or superantigen on their surface has been analyzed through the study of a specific structure named immunological synapse (IS) that serves the purpose of allowing cell-cell communication. The IS is a polarized, highly organized molecular structure that has been defined by the dynamic exclusion or accumulation of different molecules during its maturation (Sancho et al., 2002). In the classical T-B IS, a mature synapse is formed by the concentration of $\mathrm{T}$ cell receptor (TCR) complexes at the center of the structure (cSMAC), surrounded by a ring of adhesion molecules (pSMAC, see Figure 1; Vicente-Manzanares and Sanchez-Madrid, 2004). More recently, the visualization of the TCR complex dynamics at the cell surface during T cell spreading over activating surfaces has allowed the identification of TCR microclusters that may account for initial signaling. The analysis of TCR activation was revitalized by the use of artificial planar lipid bilayers containing GPI-linked peptide-MHC (pMHC) molecules and adhesion molecules, such as ICAM-1 (Dustin, 2010), or activating surfaces bearing anti-CD3 and anti-CD28 antibodies (Lasserre et al., 2010), combined with new imaging technology, such as the total internal reflection fluorescence microscopy (TIRFM). Actin and tubulin cytoskeletons have been largely analyzed to study how they structure beneath the IS. Whether the molecules involved in IS formation diffuse in a random manner or there are spatiotemporal restrictions is a question that has been studied by different means. This article aims to review some of the most recent results in the field.

\section{DYNAMICS OF TCR AND SIGNALING NANO AND MICROCLUSTERS AT THE IS}

T cells can be activated by peptides presented in the context of specific major histocompatibility complex molecules (MHC), class I or II, which are recognized by the TCR and the specific coreceptor for MHC, CD8, or CD4, respectively (van der Merwe and Dushek, 2011). They could also be activated by bacterial and viral superantigens, which do not bind to the peptide-specific, hydrophobic pocket of the MHC, but show specificity for certain variable regions in the TCR $\beta$ subunit (Scherer et al., 1993).

The TCR is able to recognize the pMHC on APCs with high sensitivity. It is clear now that the same TCR may recognize different peptides with different affinities, and this is important for the process of thymic selection and for the specific response of $\mathrm{T}$ cells to self and foreign antigens (van der Merwe and Dushek, 2011). Recently, the dwell time for TCR/MHC interaction, has been shown to correlate with discrimination between low and high affinity ligands by T cells (Palmer and Naeher, 2009). These measurements have been classically performed by surface plasmon resonance with an immobilized protein on a chip and the other in solution. More recently, the proximity of the TCR to pMHC has been measured in situ with a FRET assay. The restriction in mobility imposed by the membrane affects the kinetic parameters of the interaction, resulting in faster dissociation (Huppa et al., 2010). However, the overall affinity is increased as a result of a 100 -fold enhancement in the association rate, which may derive from the existence of pre-clustered TCRs (see Figure 1). These TCR nanoclusters would facilitate a quick rebinding of a pMHC to a second 


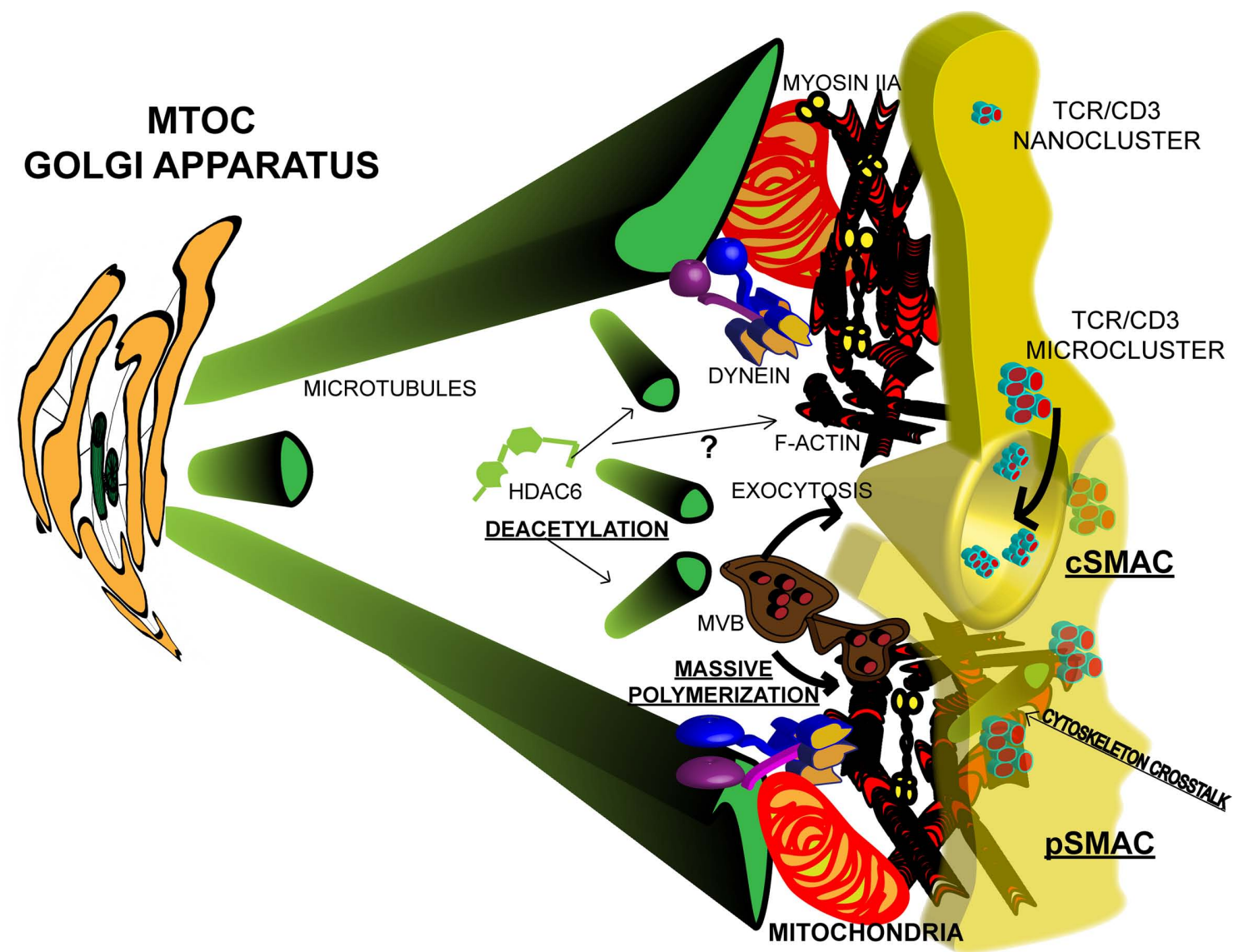

FIGURE 1 | Cytoskeletal dynamics at the Immunological synapse. TCR microclusters are formed upon activation by $\mathrm{pMHC}$ complexes, probably by fusion or apposition of pre-existing nanoclusters.

Microclusters diffuse toward the center of the IS to form the CSMAC, where they are endocytosed and recycled. TCR activation promotes the reorganization of the cytoskeleton. Tubulin dynamics are favored by deacetylation of $\alpha$-tubulin by HDAC6 and the MTOC re-orientates toward the APC through dynein/dynactin activity. The Golgi apparatus (GA) accompanies the MTOC. MVBs localize at the IS and facilitate massive polymerization of actin and exocytosis of exosomes at the immunological cleft. GA and MVBs provide the basis for polarized secretion. Mitochondria provide the energy for actomyosin contractile activity at the PSMAC. Cytoskeleton crosstalk at the IS may facilitate the movement of signaling microclusters.
TCR within the same cluster. The existence of pre-stimulated TCR nanoclusters has been shown in native gels, by electron microscopy and by confocal single molecule hsPALM microscopy (Schamel et al., 2005; Lillemeier et al., 2010).

The TCR complex includes the non-variable CD3 subunits CD3 $\varepsilon, \delta, \gamma$, and $\zeta$. All CD3 subunits contain in their cytosolic domain the so-called immunoreceptor tyrosine-based activation motifs (ITAMs) that are phosphorylated by Lck and Fyn kinases, thereby controlling the extent of TCR activation. The activation of the surface TCR is followed by a rapid conformational change in the TCR complex that allows direct Nck binding to CD $3 \varepsilon$ (Gil et al., 2002). A mutant for the CD3e ectodomain shows a dominant effect on $\mathrm{T}$ cell activation, supporting the idea that cooperative signaling between adjacent TCRs may serve to amplify TCR signals (Martinez-Martin et al., 2009). These conformational changes may be necessary for CD3 ITAM phosphorylation. In this regard, in mouse naïve $\mathrm{T}$ cells and thymocytes a constitutively active state for a significant fraction of Lck has been recently described (Nika et al., 2010), which would be ready to phosphorylate the ITAMs when a permissive conformation of the CD3 subunits is adopted. Additionally, Lck may be palmitoylated and de-palmitoylated, a manner for regulating the partitioning between cytosolic and membrane-bound fractions, which would control the proximity to substrates. A recent study through fluorescence recovery after photobleaching (FRAP) and TIRFM analyzed the differential behavior of the kinase depending on its palmitoylation state; Lck may be anchored and released from the plasma membrane within milliseconds (Zimmermann et al., 2010). Finally, a great proportion of Lck may be interacting with the co-receptor and, at least for $\mathrm{CD} 4+\mathrm{T}$ cells, this may be a form of regulating CD3 phosphorylation (Li et al., 2004).

Therefore, the pre-existing TCR nanoclusters may account for rapid microcluster formation. In this regard, TCR and LAT nanoclusters have been found in different pre-existing domains in the plasma membrane that become apposed upon TCR activation (Lillemeier et al., 2010). The microclusters are formed very fast upon TCR priming (millisecond to second), and move centripetally across the T cell/APC interface to form central cluster, 
or cSMAC, in the mature IS (see Figure 1; Mossman et al., 2005; Dustin et al., 2010). Initially, the cSMAC was described as a major site for TCR activation, due to the high concentration of TCR and associated signaling molecules, whereas the LFA-1 integrin concentrated at the periphery (Sancho et al., 2002). However, experiments demonstrating an exacerbated $\mathrm{T}$ cell activation when the formation of the CSMAC was prevented pointed to the CSMAC as a site to switch off the TCR, by becoming a site for endocytosis and recycling of the receptor (see Figure 1; Lee et al., 2002, 2003). The idea of the cSMAC being a site for TCR internalization is consistent with the presence of a strong ubiquitination signal in the cSMAC (Dustin et al., 2010), since ubiquitination has been linked to endocytosis of the receptor (Valitutti et al., 1997; Wiedemann et al., 2005). Along this line, the endosomal sorting complexes required for transport (ESCRT)-1 machinery have been shown to be necessary for correct TCR activation. The ubiquitin degradation system and $\operatorname{Tg} 101$ collaborate to the recycling of the TCR complexes. Inactivation of the system results in increased tyrosine phosphorylation (Dustin et al., 2010). The total content of tyrosine phosphorylated proteins is higher at the pSMAC than in the cSMAC when T cells are allowed to spread over activating surfaces (Mossman et al., 2005). However, in T$\mathrm{B}$ conjugates, major tyrosine phosphorylation is detected at the CSMAC upon stimulation with bacterial SAgs, whereas prevention of microtubule-organizing center (MTOC) translocation or mitochondrial re-localization at the IS makes tyrosine phosphorylated proteins to accumulate at the pSMAC (Martin-Cofreces et al., 2008; Baixauli et al., 2011). In conjugates of specific, transgenic OT-II CD4+ T cells with OVA-loaded, mature dendritic cells, either conventional or plasmacytoid, pSMAC, and cSMAC formation is also observed (Mittelbrunn et al., 2009), whereas pMHC with weaker stimulating activity do not induce formation of the cSMAC and show reduced TCR down-modulation (Dustin et al., 2010). Therefore, differences in the TCR ligands may account for spatial restrictions in protein activation at the IS in the T cell.

Other cell surface proteins, such as the co-stimulatory molecule CD28, have been shown to form microclusters at the IS. CD28 has been found to localize to the initial TCR microclusters and to co-migrate with them toward the CSMAC, where CD28 dissociates from the TCR (Yokosuka et al., 2008). Along this idea, a study on differential micropatterning with planar bilayers has shown that $\mathrm{CD} 28$ microclusters may signal at the cell periphery, promoting higher IL-2 production (Shen et al., 2008). CD28 microclusters have been found to be dependent on TCR activation and to face CD80 aggregates on the APC side in conjugates formed between dendritic cells and OT-II transgenic CD4+ T cells (Tseng et al., 2008). In human T cell/dendritic cell conjugates, class-II MHC molecules have been found to cluster in the dendritic cell, facing the TCR complex in the T cell. This highly ordered clustering was mainly dependent on ICAM1 and -3 interaction with LFA-1 in the dendritic cell and was observed more in mature than in immature dendritic cells (de la Fuente et al., 2005). LFA-1 has recently been found to form microclusters at the IS, in the so-called distal SMAC (dSMAC), which diffuse and move independently of those observed for the $\mathrm{TCR}$, although they stop migrating when they reach the pSMAC (Kaizuka et al., 2007).

\section{ROLE OF THE ACTOMYOSIN CYTOSKELETON IN ORGANIZATION OF RECEPTORS AND FUNCTION OF THE IS}

The actin cytoskeleton interconnects plasma membrane molecules with intracellular components. A massive polymerization of actin is rapidly observed at the IS, which has been related to full T cell activation (see Figure 1; Burkhardt et al., 2008; Gomez and Billadeau, 2008). However, the actin cytoskeleton also plays an important role in the destabilization of weak TCR-pMHC interactions by promoting a faster dissociation rate (Huppa et al., 2010).

Signaling by TCR microclusters might be necessary for the formation of LFA-1 microclusters through talin1, which mediates LFA- 1 clustering and the TCR-dependent increase in avidity for ICAM-1 (Simonson et al., 2006). This effect may be promoted by the participation of talin in the vinculin/talin/Arp2/3 pathway of actin polymerization and intracellular activation (Nolz et al., 2008). Whether the interaction of LFA- 1 with ICAM-3 and ICAM1 accounts for the high actin dynamics observed at the lamella is still an open question (de la Fuente et al., 2005). LFA-1 microclusters formed at the periphery of the IS have a shorter half-life than TCR microclusters. Since LFA-1 microclusters are not detected in the CSMAC, which unlike the pSMAC is very poor in polymerized actin, it is appealing to hypothesize that LFA- 1 microclusters become dispersed in the CSMAC due to their F-actin dependence (Kaizuka et al., 2007). In the pSMAC, LFA-1 microclusters may be subjected to an intense turn-over driven by myosin IIA contraction, similar to the turn-over of focal adhesions at the base of the uropod during lymphocyte migration (Sanchez-Madrid and Serrador, 2009).

Myosin IIA contractility is important for the regulation of TCR microcluster coalescence in the CSMAC, as well as for prolonged stability of the IS (see Figure 1; Ilani et al., 2009). Specific phosphorylation of the myosin light chain (MLC) at Ser19, which determines the activity of myosin IIA, depends on mitochondrial re-localization to the IS, which is driven by the mitochondrial fission factor dynamin-related protein 1 (drp1). Mitochondrial ATP is necessary for MLC phosphorylation and for the localization of the TCR at the cSMAC (Baixauli et al., 2011). Mitochondria at the IS are required to control local concentrations of calcium (Quintana et al., 2007). In drp1 knocked-down cells, total protein phosphorylation on tyrosine was found at the pSMAC instead of concentrating at the CSMAC, suggesting a mechanism for exacerbated $\mathrm{T}$ cell activation (Lee et al., 2003). These results suggest that mitochondria may serve to switch off the molecular machinery at the IS once a sufficiently sustained signal has been achieved.

\section{T CELL ACTIVATION AND ACTIN-MICROTUBULE CROSSTALK}

Microtubule-organizing center translocation is a process dependent on ITAM phosphorylation and the activity of either Lck (Sancho et al., 2002) or Fyn kinases (Martin-Cofreces et al., 2006). In resting $\mathrm{T}$ cells a fraction of Lck is located in intracellular compartments, far from the surface TCR complexes, and it is only delivered upon MTOC and Golgi apparatus translocation, in a manner dependent on the MAL cholesterol-binding proteolipid (Anton et al., 2008). The inhibition of the tubulin-based, molecular motor dynein/dynactin prevented MTOC translocation and shortened the duration of IS-emanating TCR signals 
(see Figure 1). Thus, a first signaling wave would be initiated by molecules exposed on the $\mathrm{T}$ cell surface, whereas a second wave would require the translocation of molecules such as LAT and PKC $\theta$ from intracellular compartments (Martin-Cofreces et al., 2008). MTOC translocation seems independent of mitochondrial drp1 activity. However, in dynein/dynactin defective cells neither the MTOC nor the mitochondria were found at the IS, and TCR signaling could not proceed. Of note, the TCR central cluster was not formed (Martin-Cofreces et al., 2008; Baixauli et al., 2011). Interestingly, actin polymerization was not affected even when the LFA-1 pSMAC ring was not well conformed, therefore pointing toward an effect on actomyosin dynamics rather than on actin polymerization. Dynein was proposed to have a role in the docking of microtubules emerging from the MTOC to the pSMAC, in which LFA-1 also plays an important role (Kuhn and Poenie, 2002). ADAP was also thought to connect dynein to the pSMAC (Combs et al., 2006), although it was later found that ADAP was in a peripheral ring in dynein/dynactindisrupted cells (Martin-Cofreces et al., 2008). Another role for dynein/dynactin could be the transport of transcription factors to the nucleus, since dynein/dynactin defective cells do not produce IL-2 in response to PMA/ionophore treatment (Martin-Cofreces, unpublished results). Interestingly, silencing of ezrin, another protein involved in actin polymerization and dynamics that is also linked to the tubulin cytoskeleton, has also been shown to affect IL-2 production after PMA/ionophore treatment (Lasserre et al., 2010). Ezrin knockdown disturbs the tubulin cytoskeleton and T cell signaling (Roumier et al., 2001; Lasserre et al., 2010). Ezrin was found to interact with the Disc Large 1 protein (Dlg1), which is involved in cell polarity events and in IS formation (Ludford-Menting et al., 2005), perhaps by facilitating the binding of ezrin to microtubules at the IS. Ezrin silencing disturbed the dynamics of SLP76-based microclusters, leading to increased tyrosine phosphorylation at the IS and slowed Erk1/2 inactivation (Lasserre et al., 2010).

The number of proteins that affect both cytoskeletons is growing and highlights the enormous importance of their intercommunication. AKAP450, an A-kinase anchoring protein that can be found at the MTOC in T cells, is essential for pSMAC localization and activation of LFA-1. Indeed, in AKAP450 knocked-down cells the TCR was not well recruited to the cSMAC or activated upon antigen or bacterial SAg stimulation. In addition, MTOC translocation was prevented and actin polymerization defective, but Erk1/2 activation was increased (Robles-Valero et al., 2010). This study points out to the importance of initial signaling for the reorganization of both cytoskeletons, which in turn are required to lower some pathways, as the one leading to Erk1/2 activation. The histone deacetylase 6 (HDAC6) was shown to regulate microtubule dynamics at short times upon TCR triggering in cell conjugates, in a catalytic activity-dependent manner (see Figure 1; Serrador et al., 2004). MTOC translocation and TCR clustering at the cSMAC were prevented by over-expression of HDAC6, adding further support to a role for microtubules in $\mathrm{T}$ cell activation. Indeed, the immunoprecipitation of tubulin in non-depolymerizing conditions revealed that phosphorylated CD3 $\zeta$ and ZAP70 co-precipitate with tubulin upon TCR stimulation. These data agree with the finding that signaling microclusters co-localize with microtubules and lose their localization when the tubulin cytoskeleton is distorted (Lasserre et al., 2010). Indeed, the DIA1 and FMN-L members of the formin family mediate MTOC translocation during $\mathrm{T}$ cell activation and affect the actin polymerization (Gomez and Billadeau, 2008). HS1, the homologous protein of cortactin in lymphocytes, regulates actin polymerization in T cells (Gomez and Billadeau, 2008). HDAC6 may deacetylate cortactin during cell migration, regulating the formation of the leading-edge lamella (Valenzuela-Fernandez et al., 2008). Therefore, it is possible that HDAC6 regulates the dynamics of the lamella surrounding the IS, and at the same time favors a more dynamic tubulin cytoskeleton through tubulin deacetylation (see Figure 1). HDAC6 transports misfolded proteins to the aggresome, a system dependent of ubiquitination, helped by the dynein motor, (Kawaguchi et al., 2003). As commented before, ubiquitination and degradation are important to turn off $\mathrm{T}$ cell activation. In this process, HDAC6 and dynein/dynactin might play a kind of qualitycontrol by promoting autophagy. The localization and behavior of TCR microclusters are thus dependent on whether the actomyosin and tubulin cytoskeletons reorganize correctly upon TCR stimulation, creating a positive feedback loop, since TCR activation is necessary to initiate this remodeling. On the other hand, they are also necessary to switch off signaling. Although this can appear controversial, there is a spatiotemporal regulation of cytoskeleton dynamics and it may be that the first wave of activation stimulates cytoskeletal remodeling (massive actin polymerization and MTOC translocation), leading to a second wave of stimulation that will in turn promote the activity of the cytoskeleton to switch off signaling (through actomyosin contractility and microtubule docking at the pSMAC).

\section{THE IS AS A PLATFORM FOR CELLULAR INTERCOMMUNICATION}

The secretory function of the IS has been largely analyzed in terms of effector functions, such as cytolysis of the target cell by cytotoxic T lymphocytes (CTLs) and polarized secretion of cytokines by helper T cells (Sancho et al., 2002; Huse et al., 2008). CTLs have been shown to secrete cytokines in addition to granzymes and perforin to the target cell, depending on the threshold for activation (Faroudi et al., 2003). In addition, CTLs may also polarize their lytic granules toward a CD8 $+\mathrm{T}$ cell in a Lck-dependent process once the pMHC-I from the CTL interacts with the CD8 co-receptor, thus becoming a target for the CTL (Milstein et al., 2011). This can cause the suppression of different immune responses and support the idea of different methods of cell-cell communication in the immune system, which involves reciprocal interchange of information. In this regard, the dSMAC, which is formed outside the pSMAC and is part of the high motile lamella that embraces the APC (Burkhardt et al., 2008) can be important for the maintenance of a specific gap between the T cell and the APC to promote specific secretion.

Clathrin has been shown to account for the massive actin polymerization occurring at the IS. Moreover, the subset of clathrin accounting for the massive actin polymerization at the IS is associated with multivesicular bodies (MVB) through HRS protein 
(Calabia-Linares et al., 2011), which translocates to the IS, providing an important compartment for exocytosis at the IS (see Figure 1). In fact, a specific transfer of exosomes through the IS has been recently described, which is related to MVB localization (see Figure 1; Mittelbrunn et al., 2011). These exosomes transfer specific miRNAs to the APC that can affect APC's biology. The analysis of their role on the corresponding APC deserves future research, but highlights the importance of the cSMAC both as an endocytic and exocytic focal site toward the APC. Whether the formation of MHC-II clusters at the APC side in response to the TCR activation and IS formation (de la Fuente et al., 2005)

\section{REFERENCES}

Anton, O., Batista, A., Millan, J., AndresDelgado, L., Puertollano, R., Correas, I., and Alonso, M. A. (2008). An essential role for the MAL protein in targeting Lck to the plasma membrane of human T lymphocytes. J. Exp. Med. 205, 3201-3213.

Baixauli, F., Martin-Cofreces, N. B., Morlino, G., Carrasco, Y. R., CalabiaLinares, C., Veiga, E., Serrador, J. M., and Sanchez-Madrid, F. (2011). The mitochondrial fission factor dynamin-related protein 1 modulates $\mathrm{T}$-cell receptor signalling at the immune synapse. EMBO J. 30, 1238-1250.

Burkhardt, J. K., Carrizosa, E., and Shaffer, M. H. (2008). The actin cytoskeleton in $\mathrm{T}$ cell activation. Annu. Rev. Immunol. 26, 233-259.

Calabia-Linares, C., Robles-Valero, J., De La Fuente, H., Perez-Martínez, M., Martin-Cofreces, N. B., AlfonsoPérez, M., Gutierrez-Vázquez, C., Mittelbrunn, M., Ibiza, S., UrbanoOlmos, F. R., Aguado-Ballano, C., Sánchez-Sorzano, C. O., SánchezMadrid, F., and Veiga, E. (2011). Endosomal clathrin drives actin accumulation at the immunological synapse. J. Cell Sci. 124, 820-830.

Combs, J., Kim, S. J., Tan, S., Ligon, L. A., Holzbaur, E. L., Kuhn, J., and Poenie, M. (2006). Recruitment of dynein to the Jurkat immunological synapse. Proc. Natl. Acad. Sci. U.S.A. 103, 14883-14888.

de la Fuente, H., Mittelbrunn, M., Sanchez-Martin, L., VicenteManzanares, M., Lamana, A., Pardi, R., Cabanas, C., and SanchezMadrid, F. (2005). Synaptic clusters of MHC class II molecules induced on DCs by adhesion moleculemediated initial T-cell scanning. Mol. Biol. Cell 16, 3314-3322.

Dustin, M. L. (2010). Insights into function of the immunological synapse from studies with supported planar bilayers. Curr. Top. Microbiol. Immunol. 340, 1-24.

Dustin, M. L., Chakraborty, A. K., and Shaw, A. S. (2010). Understanding the structure and function of the immunological synapse. Cold Spring Harb. Perspect. Biol. 2, a002311.

Faroudi, M., Utzny, C., Salio, M., Cerundolo, V., Guiraud, M., Muller, S., and Valitutti, S. (2003). Lytic versus stimulatory synapse in cytotoxic T lymphocyte/target cell interaction: manifestation of a dual activation threshold. Proc. Natl. Acad. Sci. U.S.A. 100, 14145-14150.

Gil, D., Schamel, W. W., Montoya, M., Sanchez-Madrid, F., and Alarcon, B. (2002). Recruitment of Nck by CD3 epsilon reveals a ligand-induced conformational change essential for $\mathrm{T}$ cell receptor signaling and synapse formation. Cell 109, 901-912.

Gomez, T. S., and Billadeau, D. D. (2008). T cell activation and the cytoskeleton: you can't have one without the other. Adv. Immunol. 97, $1-64$.

Huppa, J. B., Axmann, M., Mortelmaier, M. A., Lillemeier, B. F., Newell, E. W., Brameshuber, M., Klein, L. O., Schutz, G. J., and Davis, M. M. (2010). TCR-peptide-MHC interactions in situ show accelerated kinetics and increased affinity. Nature 463, 963-967.

Huse, M., Quann, E. J., and Davis, M. M. (2008). Shouts, whispers and the kiss of death: directional secretion in T cells. Nat. Immunol. 9, 1105-1111. Ilani, T., Vasiliver-Shamis, G., Vardhana, S., Bretscher, A., and Dustin, M. L. (2009). T cell antigen receptor signaling and immunological synapse stability require myosin IIA. Nat. Immunol. 10, 531-539.

Kaizuka, Y., Douglass, A. D., Varma, R., Dustin, M. L., and Vale, R. D. (2007). Mechanisms for segregating $\mathrm{T}$ cell receptor and adhesion molecules during immunological synapse formation in Jurkat T cells. Proc. Natl. Acad. Sci. U.S.A. 104, 20296-20301.

Kawaguchi, Y., Kovacs, J. J., McLaurin, A., Vance, J. M., Ito, A., and Yao, T. P. (2003). The deacetylase HDAC6 regulates aggresome formation and cell viability in response to misfolded protein stress. Cell 115, 727-738.

Kuhn, J. R., and Poenie, M. (2002). Dynamic polarization of the

are important to form an endocytic focus at the APC is also an interesting point to be investigated.

\section{ACKNOWLEDGMENTS}

We thank M. Gomez for editorial support of the manuscript. This work was supported by grants from Spanish Ministry of Science and Innovation (SAF2008-02635), INSINET 0159/2006 from Comunidad de Madrid, Red RECAVA RD06/0014-0030, and the Centro Nacional de Investigaciones Cardiovasculares (CNIC, Spain), which is supported by the Spanish Ministry of Science and Innovation and the Pro-CNIC Foundation.

microtubule cytoskeleton during CTL-mediated killing. Immunity 16, 111-121.

Lasserre, R., Charrin, S., Cuche, C., Danckaert, A., Thoulouze, M. I., de Chaumont, F., Duong, T., Perrault, N., Varin-Blank, N., Olivo-Marin, J. C., Etienne-Manneville, S., Arpin, M., Di Bartolo, V., and Alcover, A. (2010). Ezrin tunes T-cell activation by controlling Dlg1 and microtubule positioning at the immunological synapse. EMBO J. 29, 2301-2314.

Lee, K. H., Dinner, A. R., Tu, C., Campi, G., Raychaudhuri, S., Varma, R., Sims, T. N., Burack, W. R., Wu, H., Wang, J., Kanagawa, O., Markiewicz, M., Allen, P. M., Dustin, M. L., Chakraborty, A. K., and Shaw, A. S. (2003). The immunological synapse balances $\mathrm{T}$ cell receptor signaling and degradation. Science 302, 1218-1222.

Lee, K. H., Holdorf, A. D., Dustin, M. L., Chan, A. C., Allen, P. M., and Shaw, A. S. (2002). T cell receptor signaling precedes immunological synapse formation. Science 295, 1539-1542.

Li, Q. J., Dinner, A. R., Qi, S., Irvine, D. J., Huppa, J. B., Davis, M. M., and Chakraborty, A. K. (2004). CD4 enhances $\mathrm{T}$ cell sensitivity to antigen by coordinating Lck accumulation at the immunological synapse. Nat. Immunol. 5, 791-799.

Lillemeier, B. F., Mortelmaier, M. A., Forstner, M. B., Huppa, J. B., Groves, J. T., and Davis, M. M. (2010). TCR and Lat are expressed on separate protein islands on $\mathrm{T}$ cell membranes and concatenate during activation. Nat. Immunol. 11, 90-96.

Ludford-Menting, M. J., Oliaro, J., Sacirbegovic, F., Cheah, E. T., Pedersen, N., Thomas, S. J., Pasam, A., Iazzolino, R., Dow, L. E., Waterhouse, N. J., Murphy, A., Ellis, S., Smyth, M. J., Kershaw, M. H., Darcy, P. K., Humbert, P. O., and Russell, S. M. (2005). A network of PDZ-containing proteins regulates $\mathrm{T}$ cell polarity and morphology during migration and immunological synapse formation. Immunity 22, 737-748.
Martin-Cofreces, N. B., Robles-Valero, J., Cabrero, J. R., Mittelbrunn, M., Gordon-Alonso, M., Sung, C. H., Alarcon, B., Vazquez, J., and SanchezMadrid, F. (2008). MTOC translocation modulates IS formation and controls sustained T cell signaling. J. Cell Biol. 182, 951-962.

Martin-Cofreces, N. B., Sancho, D., Fernandez, E., Vicente-Manzanares, M. Gordon-Alonso, M., Montoya, M. C., Michel, F., Acuto, O., Alarcon, B., and Sanchez-Madrid, F. (2006). Role of Fyn in the rearrangement of tubulin cytoskeleton induced through TCR. J. Immunol. 176, 4201-4207.

Martinez-Martin, N., Risueno, R. M. Morreale, A., Zaldivar, I., FernandezArenas, E., Herranz, F., Ortiz, A. R., and Alarcon, B. (2009). Cooperativity between $\mathrm{T}$ cell receptor complexes revealed by conformational mutants of CD3epsilon. Sci. Signal. 2, ra43.

Milstein, O., Hagin, D., Lask, A., ReichZeliger, S., Shezen, E., Ophir, E., Eidelstein, Y., Afik, R., Antebi, Y. E., Dustin, M. L., and Reisner, Y. (2011). CTLs respond with activation and granule secretion when serving as targets for T-cell recognition. Blood 117, 1042-1052.

Mittelbrunn, M., Gutierrez-Vazquez, C. Villarroya-Beltri, C., Gonzalez, S., Sanchez-Cabo, F., Gonzalez, M. A., Bernad, A., and Sanchez-Madrid, F. (2011). Unidirectional transfer of microRNA-loaded exosomes from $\mathrm{T}$ cells to antigen-presenting cells. Nat. Commun. 2, 282.

Mittelbrunn, M., Martinez del Hoyo, G., Lopez-Bravo, M., Martin-Cofreces, N. B., Scholer, A., Hugues, S., Fetler, L., Amigorena, S., Ardavin, C., and Sanchez-Madrid, F. (2009). Imaging of plasmacytoid dendritic cell interactions with T cells. Blood 113, 75-84.

Mossman, K. D., Campi, G., Groves, J. T., and Dustin, M. L. (2005). Altered TCR signaling from geometrically repatterned immunological synapses. Science 310 , 1191-1193. 
Nika, K., Soldani, C., Salek, M., Paster, W., Gray, A., Etzensperger, R., Fugger, L., Polzella, P., Cerundolo, V., Dushek, O., Hofer, T., Viola, A., and Acuto, O. (2010). Constitutively active Lck kinase in $\mathrm{T}$ cells drives antigen receptor signal transduction. Immunity 32, 766-777.

Nolz, J. C., Nacusi, L. P., Segovis, C. M., Medeiros, R. B., Mitchell, J. S., Shimizu, Y., and Billadeau, D. D. (2008). The WAVE2 complex regulates $\mathrm{T}$ cell receptor signaling to integrins via Abl- and CrkL-C3Gmediated activation of Rap1. J. Cell Biol. 182, 1231-1244.

Palmer, E., and Naeher, D. (2009). Affinity threshold for thymic selection through a T-cell receptor-coreceptor zipper. Nat. Rev. Immunol. 9, 207-213.

Quintana, A., Schwindling, C., Wenning, A. S., Becherer, U., Rettig, J., Schwarz, E. C., and Hoth, M. (2007). $\mathrm{T}$ cell activation requires mitochondrial translocation to the immunological synapse. Proc. Natl. Acad. Sci. U.S.A. 104, 14418-14423.

Robles-Valero, J., Martin-Cofreces, N. B., Lamana, A., Macdonald, S., Volkov, Y., and Sanchez-Madrid, F. (2010). Integrin and CD3/TCR activation are regulated by the scaffold protein AKAP450. Blood 115, 4174-4184.

Roumier, A., Olivo-Marin, J. C., Arpin, M., Michel, F., Martin, M., Mangeat, P., Acuto, O., Dautry-Varsat, A., and Alcover, A. (2001). The membrane-microfilament linker ezrin is involved in the formation of the immunological synapse and in $\mathrm{T}$ cell activation. Immunity 15 , 715-728.

Sanchez-Madrid, F., and Serrador, J. M. (2009). Bringing up the rear: defining the roles of the uropod. Nat. Rev. Mol. Cell Biol. 10, 353-359.

Sancho, D., Vicente-Manzanares, M., Mittelbrunn, M., Montoya, M. C., Gordon-Alonso, M., Serrador, J. M., and Sanchez-Madrid, F. (2002). Regulation of microtubule-organizing center orientation and actomyosin cytoskeleton rearrangement during immune interactions. Immunol. Rev. 189, 84-97.

Schamel, W. W., Arechaga, I., Risueno, R. M., van Santen, H. M., Cabezas, P., Risco, C., Valpuesta, J. M., and Alarcon, B. (2005). Coexistence of multivalent and monovalent TCRs explains high sensitivity and wide range of response. J. Exp. Med. 202, 493-503.

Scherer, M. T., Ignatowicz, L., Winslow, G. M., Kappler, J. W., and Marrack, P. (1993). Superantigens: bacterial and viral proteins that manipulate the immune system. Annu. Rev. Cell Biol. 9, 101-128.

Serrador, J. M., Cabrero, J. R., Sancho, D., Mittelbrunn, M., Urzainqui, A., and Sanchez-Madrid, F. (2004). HDAC6 deacetylase activity links the tubulin cytoskeleton with immune synapse organization. Immunity 20, 417-428.

Shen, K., Thomas, V. K., Dustin, M. L., and Kam, L. C. (2008). Micropatterning of costimulatory ligands enhances CD4+ $\mathrm{T}$ cell function. Proc. Natl. Acad. Sci. U.S.A. 105, 7791-7796.
Simonson, W. T., Franco, S. J., and Huttenlocher, A. (2006). Talin1 regulates TCR-mediated LFA-1 function. J. Immunol. 177, 7707-7714.

Tseng, S. Y., Waite, J. C., Liu, M., Vardhana, S., and Dustin, M. L. (2008). $\mathrm{T}$ cell-dendritic cell immunological synapses contain TCR-dependent CD28-CD80 clusters that recruit protein kinase C theta. J. Immunol. 181, 4852-4863.

Valenzuela-Fernandez, A., Cabrero, J. R., Serrador, J. M., and SanchezMadrid, F. (2008). HDAC6: a key regulator of cytoskeleton, cell migration and cell-cell interactions. Trends Cell Biol. 18, 291-297.

Valitutti, S., Muller, S., Salio, M., and Lanzavecchia, A. (1997). Degradation of $\mathrm{T}$ cell receptor (TCR)CD3-zeta complexes after antigenic stimulation. J. Exp. Med. 185, 1859-1864.

van der Merwe, P. A., and Dushek, O. (2011). Mechanisms for T cell receptor triggering. Nat. Rev. Immunol. 11, 47-55.

Vicente-Manzanares, M., and SanchezMadrid, F. (2004). Role of the cytoskeleton during leukocyte responses. Nat. Rev. Immunol. 4, 110-122.

Wiedemann, A., Muller, S., Favier, B., Penna, D., Guiraud, M., Delmas, C., Champagne, E., and Valitutti, S. (2005). T-cell activation is accompanied by an ubiquitination process occurring at the immunological synapse. Immunol. Lett. 98, 57-61.

Yokosuka, T., Kobayashi, W., SakataSogawa, K., Takamatsu, M., Hashimoto-Tane, A., Dustin, M. L.,
Tokunaga, M., and Saito, T. (2008). Spatiotemporal regulation of $\mathrm{T}$ cell costimulation by TCR-CD28 microclusters and protein kinase $\mathrm{C}$ theta translocation. Immunity 29, 589-601.

Zimmermann, L., Paster, W., Weghuber, J., Eckerstorfer, P., Stockinger, H., and Schutz, G. J. (2010). Direct observation and quantitative analysis of Lck exchange between plasma membrane and cytosol in living T cells. J. Biol. Chem. 285, 6063-6070.

Conflict of Interest Statement: The authors declare that the research was conducted in the absence of any commercial or financial relationships that could be construed as a potential conflict of interest.

Received: 09 April 2011; accepted: 15 June 2011; published online: 04 July 2011.

Citation: Martín-Cófreces NB, Alarcón B and Sánchez-Madrid F (2011) Tubulin and actin interplay at the $T$ cell and antigen-presenting cell interface. Front. Immun. 2:24. doi: 10.3389/fimmu.2011.00024

This article was submitted to Frontiers in $T$ Cell Biology, a specialty of Frontiers in Immunology.

Copyright (C) 2011 Martín-Cófreces, Alarcón and Sánchez-Madrid. This is an open-access article subject to a nonexclusive license between the authors and Frontiers Media SA, which permits use, distribution and reproduction in other forums, provided the original authors and source are credited and other Frontiers conditions are complied with. 